\title{
Sex-specific genetic effects associated with pigmentation, sensitivity to sunlight, and melanoma in a population of Spanish origin
}

\author{
Barbara Hernando ${ }^{1+}$, Maider Ibarrola-Villava ${ }^{2+}$, Lara P. Fernandez ${ }^{3}$, Maria Peña-Chilet ${ }^{2}$, Marta Llorca-Cardeñosa ${ }^{2}$, \\ Sara S. Oltra ${ }^{2}$, Santos Alonso ${ }^{4}$, Maria Dolores Boyano ${ }^{5,6}$, Conrado Martinez-Cadenas ${ }^{1^{*}}$ (D) and Gloria Ribas ${ }^{2^{*}}$
}

\begin{abstract}
Background: Human pigmentation is a polygenic quantitative trait with high heritability. In addition to genetic factors, it has been shown that pigmentation can be modulated by oestrogens and androgens via up- or down-regulation of melanin synthesis. Our aim was to identify possible sex differences in pigmentation phenotype as well as in melanoma association in a melanoma case-control population of Spanish origin.

Methods: Five hundred and ninety-nine females (316 melanoma cases and 283 controls) and 458 males (234 melanoma cases and 224 controls) were analysed. We genotyped 363 polymorphisms (single nucleotide polymorphisms (SNPs)) from 65 pigmentation gene regions.

Results: When samples were stratified by sex, we observed more SNPs associated with dark pigmentation and good sun tolerance in females than in males (107 versus $\left.75 ; P=2.32 \times 10^{-6}\right)$, who were instead associated with light pigmentation and poor sun tolerance. Furthermore, six SNPs in TYR, SILV/CDK2, GPR143, and F2RL1 showed strong differences in melanoma risk by sex $(P<0.01)$.
\end{abstract}

Conclusions: We demonstrate that these genetic variants are important for pigmentation as well as for melanoma risk, and also provide suggestive evidence for potential differences in genetic effects by sex.

Keywords: Pigmentation, UV sensitivity, Skin cancer, Sex, Polymorphisms

\section{Background}

Human pigmentation traits are some of the most visible and differentiable human characteristics. Pigmentation in human tissue is attributable to the number, size and cellular distribution of melanosomes produced, and the type of melanin synthesised (the black-brown coloured eumelanin or the red-yellow coloured phaeomelanin), while the number of melanocytes is usually unchanged [1].

The type of melanin synthesised is influenced by sun exposure and is genetically controlled [2]. Ultraviolet

\footnotetext{
*Correspondence: ccadenas@uji.es; gribas@incliva.es

${ }^{\dagger}$ Equal contributors

${ }^{1}$ Department of Medicine, Jaume I University of Castellon, Av. Sos Baynat s/n, 12071 Castellon, Spain

${ }^{2}$ Department of Medical Oncology, Biomedical Research Institute - INCLIVA, University of Valencia, Av. Menendez Pelayo 4 accesorio, 46010 Valencia, Spain Full list of author information is available at the end of the article
}

(UV) exposure plays a key role in the evolutionary selective pressure on human pigmentation. Geographic distribution of human skin pigmentation reflects an adaptation to latitude-dependent levels of UV radiation $[3,4]$. The linear relationship between worldwide skin pigmentation variation, latitude, and UV radiation levels is thought to result from the physiological role of melanin type in UV-mediated vitamin D synthesis, UV-induced photolysis of folate, and in the protection from exposure to UV, which can cause sunburn and skin cancer [5]. However, the physiological role for eye and hair colour variations still remains unknown.

Variation in genes implicated in human pigmentation has been associated with phenotypic characteristics such as skin colour, hair colour, eye colour, freckling, and sensitivity to sunlight [6], and also with the risk of various 
types of skin cancer [7-15]. The proteins encoded by these genes have effects at various stages of the pigmentation pathway, ranging from melanogenesis, the stabilisation and transport of enzymes in the melanin production pathway, the production and maintenance of melanosomes and the melanosomal environment, and the switch between the production of eumelanin and phaeomelanin. Other pigmentation-related proteins code for intrinsic factors that help in the regulation of pigmentation, such as factors produced by fibroblasts in the dermis that affect overlying melanocytes and keratinocytes, and endocrine factors from the blood supply, as well as neural factors and inflammation-related factors $[6,16,17]$.

Melanin synthesis is also modulated, in part, by oestrogens and androgens [18]. Physiological hyperpigmentation in various forms (tanning, dark spots, chloasma, linea nigra, and/or melasma) is commonly seen in pregnant females due to an increase of the levels of pregnancyrelated hormones [18]. The increase of pregnancy-related hormones-oestrogen, progesterone, and melanocytestimulating hormone $(\alpha-\mathrm{MSH})$-during gestation induces the activation and expression of genes involved in melanin synthesis in melanocytes [19], while it has also been shown that androgens inhibit tyrosinase activity [20]. In addition to sex-endocrine factors, the use of oestrogencontaining oral contraceptives, certain cosmetics, and oestrogen-progesterone therapies has also been associated with hyperpigmentation [21].

Biological and behavioural gender differences likely contribute to the sexual disparity in skin aging, pigmentation, and melanoma incidence and outcome [22, 23]. Recent studies point to Caucasian females having slightly darker eye colour [24, 25] and skin colour [26] than Caucasian males. Regarding melanoma, females show lower melanoma predisposition and incidence, lower risk of metastases, and longer melanoma-specific survival rates than males [27, 28]. Anatomic location of melanoma indeed tends to be different between sexes, being most commonly on the lower leg, hip, and thigh in females and on the back, abdomen, and chest in males [27].

In order to reveal possible sex-related differences in pigmentation phenotype as well as in melanoma association, we investigated the influence of 363 polymorphisms from 65 gene regions-previously associated with pigmentation traits, congenital pigmentation genetic syndromes, and/or skin cancer-in a melanoma case-control population of Spanish origin.

\section{Methods}

\section{Study subjects and data collection}

In this study, a total number of 599 females (316 melanoma cases and 283 cancer-free controls) and 458 males (234 melanoma cases and 224 cancer-free controls) were collected at several Spanish hospitals.
We carefully selected all cases and controls included in the current study to account for confounding variables. All individuals were Caucasians of Spanish origin where, according to a previous work by Laayouni and cols, there is no evidence of genetic heterogeneity within different Spanish geographical regions [29]. Controls were frequency-matched to the cases by age and place of birth.

A standardised questionnaire was used to collect information on sex, age, pigmentation characteristics (eye colour, hair colour, skin colour, number of naevi, and presence of solar lentigines), history of childhood sunburns, Fitzpatrick's skin type classification, and personal and family history of cancer, to classify individuals as previously described [30]. Forty melanoma cases from our previous work were excluded in the current analysis due to the absence of sex details.

All individuals gave written informed consent and the study was approved by the Ethics Committee of the Gregorio Marañon Hospital (Madrid, Spain) and the Biomedical Research Institute - INCLIVA (Valencia, Spain).

\section{Gene, SNP selection, and genotyping}

Gene and single nucleotide polymorphism (SNP) selection was performed as previously described [30]. Sixty-five gene regions were included in this study. They covered a broad range of biological processes, mostly related to pigmentation. We genotyped a total number of 384 tag-SNPs from the selected genes ranging from the hypothetical promoter area (approximately $10 \mathrm{~kb}$ upstream) until approximately $5 \mathrm{~kb}$ downstream of the gene. SNP codes, locations, and frequencies were obtained from NCBI (www.ncbi.nlm.nih. gov/SNP), HapMap (www.hapmap.org), and Illumina databases. A minor allele frequency (MAF) threshold of 0.05 in the HapMap CEU population and an 'Illumina score' not lower than 0.6 (as recommended by manufacturer) were established to ensure high genotyping success rate of the SNPs selected.

SNP genotyping was done using the Golden Gate Assay according to manufacturer's protocol (Illumina, San Diego, CA, USA), as previously described [30].

\section{Statistical analysis}

Quality control processes and allelic and genotypic association tests were performed using the SNPator software (www.snpator.com). Additional statistical analyses and plots were conducted using the $\mathrm{R}$ statistical framework. All genetic analyses were performed estimating the effect of the minor allele in the Spanish population.

For all polymorphisms studied, Fisher's exact test was used both to test for deviations from Hardy-Weinberg equilibrium (HWE) between sexes and to compare allele counts between female and male individuals. Bonferroni 
correction was applied and $P$ values higher than $1.37 \times 10^{-4}$ were considered in HWE.

Associations between the genotyped SNPs and various pigmentation and sun sensitivity traits were assessed via logistic regression, coded additively for each copy of the minor allele. This was done for males and females separately, with eye colour (blue/green versus brown/black), hair colour (brown/black versus blond/red), skin colour (fair versus dark), number of naevi $(\geq 50$ versus $<50$ ), presence of lentigines (yes versus no), and childhood sunburn (yes versus no) as the outcome variables. Genotyperelated Odds Ratios (ORs), their corresponding $95 \%$ confidence intervals $(\mathrm{CIs})$ and associated $P$ values were estimated. Results of the association analysis were represented using volcano plots, mapping significance $(-\log 10 P$ value) versus fold-change $(\log 2 \mathrm{OR})$ for the comparison between individuals for eye colour, hair colour, skin colour, presence of lentigines, childhood sunburns and naevi number separately. $P$ values were two sided and those lower than 0.01 were considered statistically significant (since six pigmentation traits were studied separately, statistical significant threshold of $P$ value $<0.05 / 6=0.01$ )

In order to have an overview of all the significant estimates obtained in the sex-specific logistic regression analyses, we evaluated the differences in the number of polymorphisms associated both with protective and risk phenotypes between sex groups $(P$ values $<0.05)$, using $2 \times 2$ contingency tables and performing a Fisher's exact test.

Logistic regression was performed to re-assess associations between genotypes and melanoma risk done previously [30], but separating individuals by sex in order to estimate sex-specific ORs, $95 \%$ CIs and $P$ values. As mentioned above, the minor allele was also modelled under an additive model. Using the same criteria as in the analysis of pigmentation traits, two-sided $P$ values lower than 0.01 were considered to constitute evidence of association.

Finally, we performed a sex-differentiated regression estimate test for each SNP for all phenotypic traits. We tested for equality of sex-specific allelic effects with the aim of obtaining sex-differentiated $P$ values [31], and a statistical significance threshold of sex-differentiated $P$ value $<0.05$ was used. Briefly, for each sex-specific association test, sex-specific beta coefficients (log ORs) and the corresponding standard errors were evaluated using a Chi-square test with one degree of freedom. Then, a Chi-square test with two degrees of freedom was performed for each SNP, in which the previously calculated female-specific and male-specific Chi-square statistics were added up. Finally, a test of heterogeneity of allelic effects between males and females using a Chi-square test with one degree of freedom was conducted. A significant sex-specific and sex-differentiated $P$ value is required to verify a potential significance in allelic effect by sex, following the same criteria used by Kocarnik and cols. [32]. Manhattan plots were used to display the strength of significant differences between male-only and female-only associated effects $(-\log 10$ sex-differentiated $P$ value) for each trait tested.

\section{Results and discussion}

Our sample set included 599 females and 458 males of Spanish ancestry. Median age was 45 years (range 18-92) for females and 47 years (range 18-92) for males. Regarding control individuals, mean age was 42 years (range 18-91) for females and 45 years (range 18-90) for males. Melanoma cases presented a median age of 52 years (range 18-92) for females and 53 years (range 18-92) for males. Since age differences were not observed between sample subsets $(P$ value $>0.05)$, association analyses were not adjusted by age.

From an initial list of 384 tag-SNPs selected, 21 SNPs (5.4\%) were discarded due to failed genotyping (no PCR amplification, insufficient intensity for cluster separation or poor cluster definition). All 363 remaining SNPs were in HWE after Bonferroni correction (Additional file 1: Table S1). Minor allele frequencies estimated for each SNP were almost identical for females and males, with a remarkable linear correlation $\left(R^{2}\right)$ of 0.982 (Additional file 1: Figure S1).

\section{Association with phenotypic characteristics by sex}

In a previous study published by our group, the association of some genes with phenotypic characteristics was reported [30]. However, analyses were performed without taking into account sex data. In the current study, samples were additionally stratified by sex to evaluate differences in pigmentation and sun response between males and females.

Thirty four SNPs showed association with at least one pigmentation trait, and 42 SNPs were associated with at least one sun response trait studied $(P<0.01)$ (Additional file 1: Tables S2 and S3). Each of these polymorphisms displayed a moderate effect on pigmentation in our Spanish population dataset. Our results showed apparent differences in the direction of the association with the pigmentation characteristics, with variants showing ORs below 1.0 correlated with dark pigmentation and/or good tolerance to sunlight, and variants with ORs above 1.0 associated with light pigmentation and/or poor tolerance to sunlight. Variants in these genes most likely play important roles in the differences in pigmentation and tanning response among individuals of the Spanish population, and subsequently also in skin cancer risk [33].

Representations of $-\log 10 P$ values versus $\log 2$ ORs comparing 599 female individuals to 458 males for eye 
colour, hair colour, skin colour, presence of lentigines, childhood sunburns and naevi number are shown in Fig. 1. Detailed information on rs numbers, genes, chromosome locations, minor alleles, ORs, $95 \%$ CIs, and $P$ values for pigmentation and sun response characteristics are summarised in Additional file 1: Tables S2 and S3.

Sex-specific analyses in this study showed significant differences in the pattern of association with pigmentation and tanning response traits between male and female individuals. Out of all SNPs with significant sex-specific associations, we found significantly more SNPs associated with dark pigmentation or sun protection in female than in male individuals (107 versus $75 ; P=2.32 \times 10^{-6}$ ), the latter being more commonly associated with light pigmentation and poor sun tolerance traits highly associated with melanoma predisposition [9] (Fig. 2). The percentage of SNPs associated with both dark eye and dark hair colour in females was higher than in males $(72.72$ versus $40.74 \%, P=0.025 ; 78.57$ versus $48.28 \%, P=0.018$, respectively). This association pattern was also observed for skin colour, but without significance (66.67 versus $41.94 \%, P=0.068)$. In addition, female individuals presented more SNPs associated with both $\leq 50$ naevi and absence of childhood sunburns than males (65.38 versus 36.67, $P=0.032$; 61.11 versus $36.11 \%, P=0.034$; respectively). On the other hand, a similar percentage of SNPs associated with absence of lentigines was observed in both female and male individuals (56.00 versus $43.33 \%, P=0.35$ ). A representation of the distribution/count of polymorphisms associated with phenotype groups for each trait studied, separated by sex, is displayed in Fig. 2 .

It is important to note that these associations do not reflect differences in the allelic frequencies of these pigmentation genes between males and females. These results basically indicate that, for a given genotype, the allelic effects on the phenotypic traits are shown to be significantly different in both sexes.

Additionally, sex-differentiated analysis was performed in order to test for equality between male-specific and female-specific regression estimates. Sex-differentiated $P$ values are represented in Fig. 3. A significant sex-specific and sex-differentiated SNP association is required to establish a potential difference in effect for each polymorphism

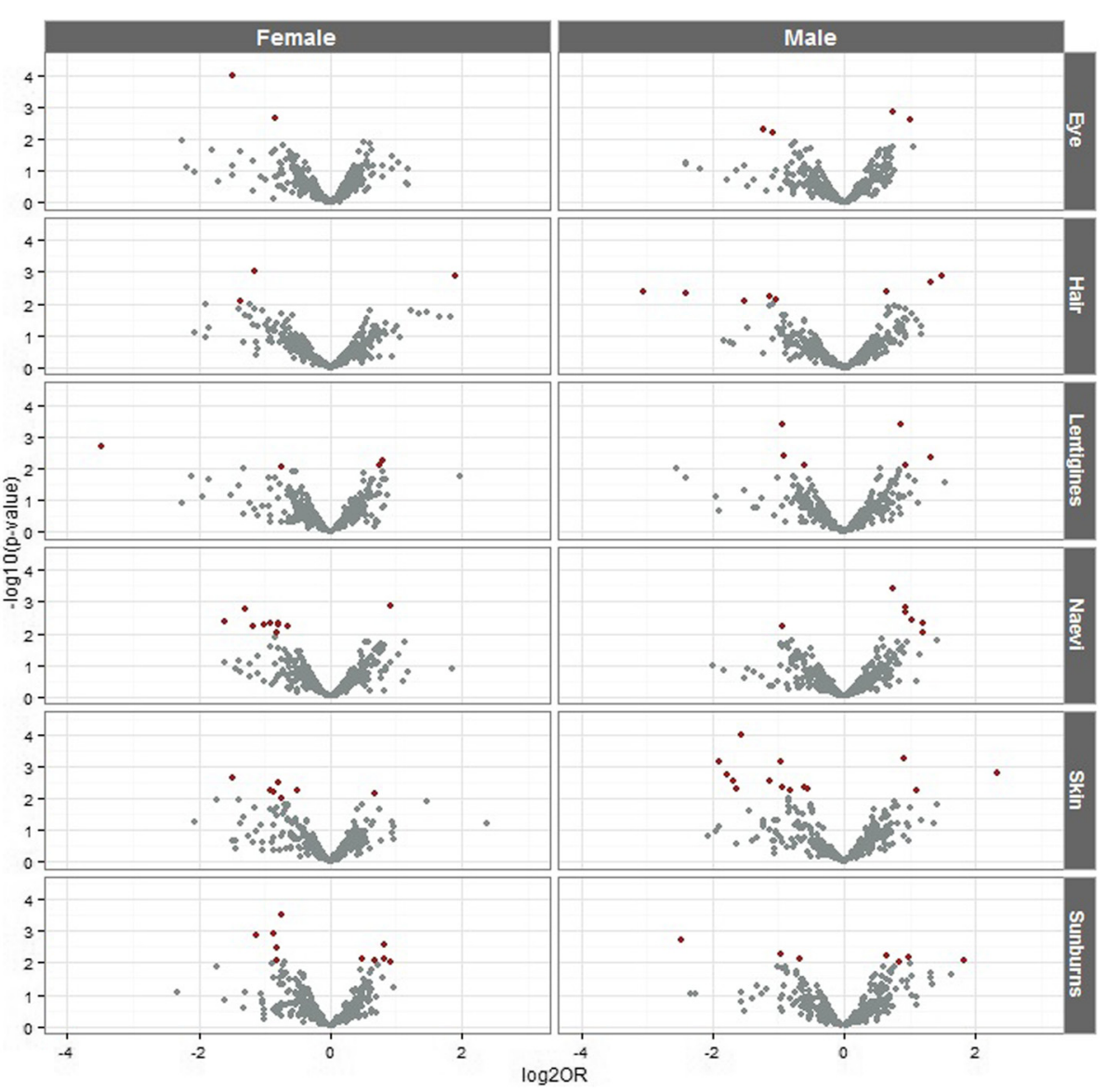

Fig. 1 Volcano plots showing significance $(-\log 10 P$ value) versus fold change (log2 OR) for pigmentation and sun sensitivity traits separated by sex. Red dots indicate SNPs with a significant fold change $(P$ values $<0.01)$ 


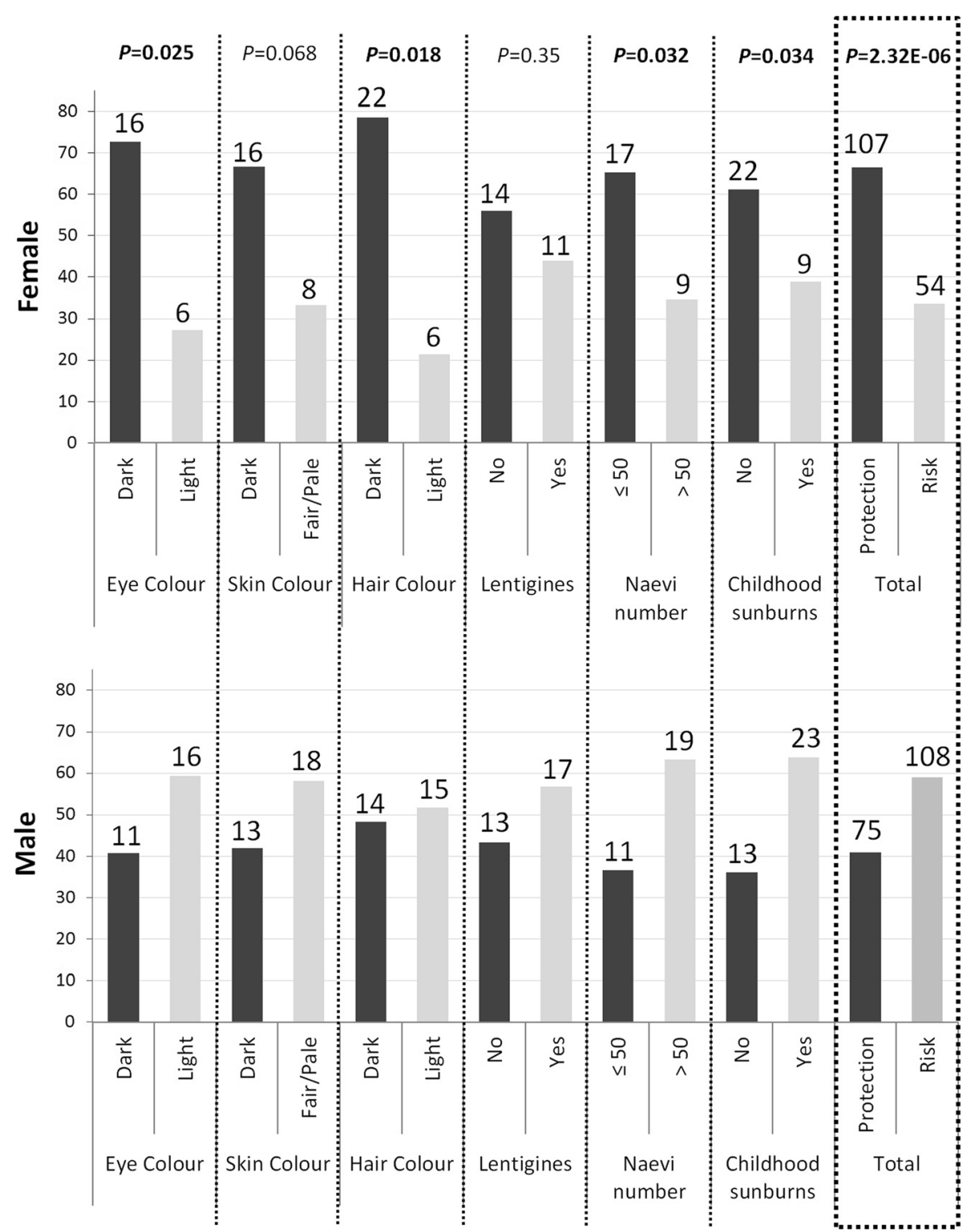

Fig. 2 Distribution of the SNPs associated with pigmentation and sun sensitivity traits separated by sex. The percentage of each phenotype (protection or risk) is calculated taking into account the total number of significant SNPs associated in males and females $(P$ values $<0.05)$. Percentages are represented by bars of the corresponding colour. The number on the top of each bar represents the count of associated SNPs in each category

by sex. Three SNPs showed a strong potential sexdifference in eye colour effect, 10 SNPs in skin colour effect, 3 SNPs in hair colour effect, 4 SNPs in sunburns effect, 5 SNPs in lentigines effect, and 5 SNPs in naevi effect $(P<0.01)$. Among these SNPs, PLDN SNP rs12909221, GPR143 SNP rs2521667, POMC SNP rs6734859, AP3M2 SNP rs7009632, BCL2 SNP rs1462129, and TYRP1 SNP rs10809828 were associated with light pigmentation and poor sun tolerance in males. Only one polymorphism, rs2521578 on the GPR143 gene, showed a high association with poor sun tolerance in females (Additional file 1: Tables S2 and S3).
Promising differences in allelic effect by sex were also observed for TYR SNP rs1042602. Females and males showed statistically significant effects in opposite directions for this SNP, and this difference in effect by sex would remain hard to discriminate from chance. Indeed, a sex-differentiated $P$ value of $1.30 \times 10^{-3}$ was estimated for rs1042602, as shown in Fig. 3.

Polymorphisms showing potential differences in allelic effect by sex are located on genes that have functions related to melanocyte development, melanosome formation, maturation, and transportation, as well as to skin cancer $[6,9,10,15,16,30,34-37]$. Interestingly, we also found 


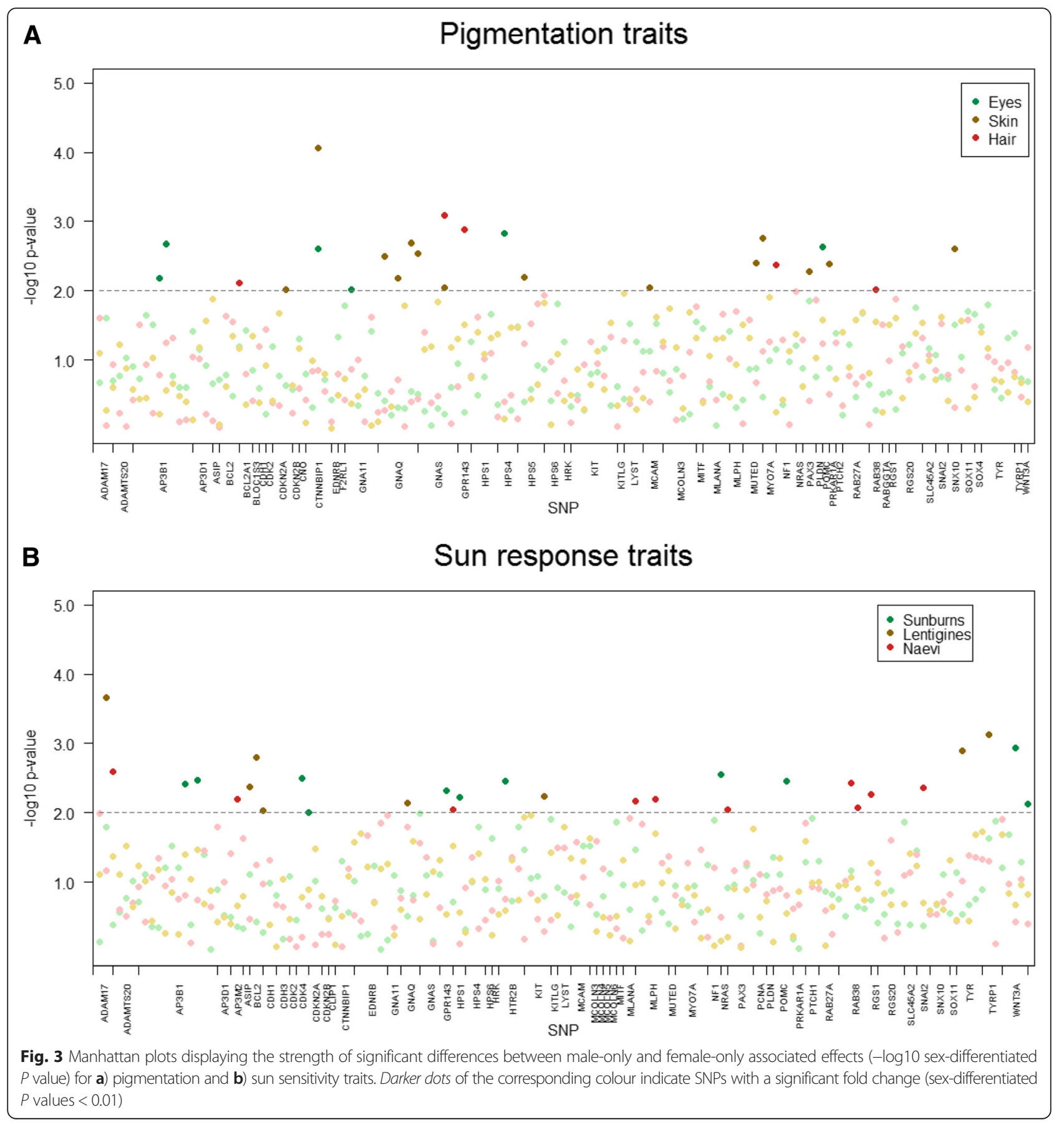

associations between pigmentation phenotypes and several genes-CDKN2A, GNA11, NRAS, and WNT3A-involved in the up-regulation of melanogenic genes, the activation, survival, and proliferation of the melanocyte, and/or the processes leading to carcinogenesis.

\section{Associations with melanoma risk by sex}

In a previous study published by our group, the association of 65 gene regions with melanoma risk was reported [30]. However, at that time, no sex stratification was applied to perform the association analysis. In this work, we have carried out an analysis of association between genotypes and melanoma risk for female and male individuals separately.

Sixteen SNPs located in 10 genes showed consistent male- or female-specific association with melanoma risk. Eleven of those SNPs showed potential differences in effect by sex, since $P$ values obtained in the sex-differentiated regression estimate test were lower than 0.05 . Detailed information on rs numbers, genes, chromosome locations, 
minor alleles, ORs, $95 \%$ CIs, and $P$ values for melanoma risk are summarised in Table 1.

Among these 11 SNPs, we found six SNPs located in 4 genes showing a strong difference in melanoma risk effect when samples were stratified by sex - sex-specific and sex-differentiated $P$ values lower than 0.01 . F2RL1 SNP rs2242991, GPR143 SNPs rs2521667, and rs2732872, and TYR SNP rs5021654 increased melanoma predisposition in males as opposed to females. Additionally, a strong melanoma protective effect was displayed by rs2069398 on CDK2/SILV in females only. These SNPs were also associated with pigmentation and sun tolerance in opposite directions in males (ORs $>1$, melanoma risk traits) versus females (ORs $<1$, melanoma protective traits), supporting lower melanoma predisposition and incidence in females than in males. Oppositely, rs1042602 on the TYR gene showed a melanoma protective effect in males compared to females. Therefore, these results are in accordance with the association between rs1042602 and dark pigmentation and good sun tolerance in males but not in females (Additional file 1: Tables S2 and S3).

These genes with potential differences in melanoma risk effect by sex are graphically represented in Additional file 1: Figure S2. The F2RL1/PAR2 gene, expressed in keratinocytes but not in melanocytes, is a G-protein coupled receptor involved in melanosome transfer [38], and changes in its expression pattern are correlated with skin cancer progression [39]. The GPR143 gene, located in the
X chromosome, encodes for a G-protein coupled receptor for tyrosine, L-DOPA, and dopamine localised on melanosomal membranes and plays an important role in melanosome biogenesis, organisation and transport. Ocular albinism type 1 (OA1; MIM300500) is caused by mutations in GPR143 and is transmitted as an Xlinked trait. The TYR gene codes for another melanosomal membrane-bound enzyme involved in the ratelimiting steps of melanogenesis. Mutations in the TYR gene are associated with light pigmentation, freckling and sun sensitivity-well-recognised melanoma risk factors-as well as with melanoma $[30,40]$. The CDK2 gene, which overlaps with the melanocyte-specific gene $\operatorname{SILV}$, is also important for melanoma growth and proliferation [41]. SILV melanosomal matrix protein represents a melanoma-specific antigen recognised by tumour infiltrating cytotoxic T lymphocytes [42].

A recent study is worth mentioning in this respect. According to Kocarnik and cols. (2014), SLC45A2 SNP rs16891982, the non-synonymous mutation F374L located in exon 5 , influenced melanoma risk differently by sex, with higher melanoma risk for males than females, probably through alterations in melanogenesis and pigmentation [32]. In our study, two SNPs on the SLC45A2 gene (rs35414 and rs35415) displayed associations with melanoma in both female-only and male-only analysis, although they do not present significant sexdifferentiated $P$ values. It is important to note that

Table 1 SNPs highly associated with melanoma risk in sex-stratified analysis

\begin{tabular}{|c|c|c|c|c|c|c|c|c|}
\hline \multirow[b]{3}{*}{ Gene } & \multirow[b]{3}{*}{ SNP ID } & \multirow[b]{3}{*}{ Chr } & \multirow[b]{3}{*}{$\mathrm{mA}$} & \multicolumn{5}{|c|}{ Melanoma } \\
\hline & & & & \multicolumn{2}{|l|}{ Female } & \multicolumn{2}{|l|}{ Male } & \multirow{2}{*}{$\begin{array}{l}\text { Sex-diff } \\
P \text { value }\end{array}$} \\
\hline & & & & $P$ value & OR & $P$ value & OR & \\
\hline$A P 3 B 1$ & rs11742673 & 5 & A & 0.0020 & $1.43(1.14-1.80)$ & 0.88 & $0.98(0.75-1.28)$ & 0.0210 \\
\hline CDK2/SILV & rs2069398 & 12 & A & $3.03 E-4$ & $0.47(0.31-0.71)$ & 0.06 & $0.60(0.35-1.03)$ & 0.0016 \\
\hline$F 2 R L 1$ & rs2242991 & 5 & G & 0.56 & $0.92(0.68-1.22)$ & 0.0076 & $1.61(1.13-2.29)$ & 0.0067 \\
\hline \multirow[t]{2}{*}{ GPR143 } & rs2521667 & $x$ & G & 0.46 & $0.89(0.67-1.20)$ & $7.04 E-4$ & $1.89(1.30-2.74)$ & $7.85 E-4$ \\
\hline & rs2732872 & $x$ & C & 0.78 & $0.96(0.73-1.27)$ & $8.43 E-4$ & $1.81(1.27-2.57)$ & 0.0052 \\
\hline KIT & rs6554198 & 4 & G & 0.46 & $0.92(0.73-1.15)$ & 0.0027 & $0.67(0.51-0.87)$ & 0.07 \\
\hline MYOTA & rs3758708 & 11 & A & 0.20 & $1.30(0.87-1.95)$ & 4. $12 E-4$ & $2.38(1.46-3.90)$ & 0.0480 \\
\hline RAB38 & rs524121 & 11 & C & 0.0086 & $0.51(0.30-0.85)$ & 0.36 & $1.32(0.73-2.38)$ & 0.0210 \\
\hline RGS20 & rs6981243 & 8 & C & 0.38 & $0.90(0.71-1.14)$ & 0.0021 & $0.66(0.51-0.86)$ & 0.0490 \\
\hline \multirow[t]{2}{*}{ SLC45A2 } & rs35414 & 5 & $\mathrm{~T}$ & 0.03 & $0.77(0.61-0.97)$ & 0.0018 & $0.66(0.51-0.85)$ & 0.39 \\
\hline & rs35415 & 5 & A & 0.03 & $0.78(0.62-0.97)$ & 0.0080 & $0.70(0.54-0.91)$ & 0.35 \\
\hline \multirow[t]{5}{*}{ TYR } & rs1042602 & 11 & A & 0.35 & $1.11(0.89-1.40)$ & 0.0047 & $0.68(0.53-0.89)$ & 0.0081 \\
\hline & rs12270717 & 11 & C & 0.60 & $1.07(0.82-1.40)$ & 0.0032 & $1.56(1.16-2.09)$ & 0.09 \\
\hline & rs17793678 & 11 & $\mathrm{~T}$ & 0.32 & $1.14(0.88-1.49)$ & 0.0036 & $1.55(1.15-2.08)$ & 0.26 \\
\hline & rs2186640 & 11 & G & 0.39 & $0.90(0.71-1.15)$ & $9.20 E-4$ & $1.57(1.20-2.05)$ & 0.0380 \\
\hline & rs5021654 & 11 & C & 0.47 & $0.92(0.72-1.16)$ & $2.03 E-4$ & $1.66(1.27-2.17)$ & 0.0014 \\
\hline
\end{tabular}


the minor allele of these two SNPs showed a protective effect for melanoma and that the allele frequencies for these protective minor alleles, causing a darker pigmentation, are actually quite common in the Spanish population, as opposed to Northern European populations. Subsequently, this association was stronger in males (rs35414: OR $=0.66,95 \%$ CI $0.51-0.85$, $P=0.0018$; and rs35415: $\mathrm{OR}=0.70,95 \% \mathrm{CI} 0.54-$ $0.91, P=0.008)$ than in females (rs35414: $\mathrm{OR}=0.77$, $95 \%$ CI $0.61-0.97, P=0.026$; and rs 35415 : $\mathrm{OR}=0.78$, $95 \%$ CI $0.62-0.97, P=0.029)$. In the male-only but not in the female-only analysis, rs35414 and rs35415 tended to be also associated with dark pigmentation and the absence of childhood sunburns $(P<0.05)$. It is important to state here that in the work by Kocarnik and cols., it was the major allele-in the Caucasian population-of the SLC45A2 SNP that was in fact modelled as the purposed risk allele for melanoma, while in this study it is the minor allele of the two SLC45A2 SNPs that was actually used as reference to perform the analyses. Therefore, the genetic effect shown by the SLC45A2 gene in our study exhibits the opposite direction that the one displayed by the Kocarnik and cols. work.

We are aware of the limitations of the current work. Firstly, the sample size was relatively restricted after dividing by sex the complete sample set, probably resulting in limited statistical power to detect modest effects for additional SNPs. Unfortunately, there are not previously published genome-wide studies presenting data stratified by sex, hindering chances of enlarging the sample size. Secondly, the subjective nature of the attributes considered may be another reason for misclassification. Thirdly, we presented two-sided unadjusted $P$ values for the associations considered; and the level of statistical significance considered was lower than the threshold required to declare unequivocally positive results. However, the results of this work-as well as other previous studies $[24,25,32]$-show that there is a strong tendency showing sex-differentiated genetic effects in pigmentary traits. Therefore, we believe that the work presented here is nonetheless reporting very interesting findings. For all these limitations, replication of our findings is essential before venturing on drawing firmer conclusions.

The results of this study suggest that there are indeed sex-specific genetic effects in human pigmentation, with larger effects for darker pigmentation in females compared to males. A plausible cause might be the differentially expressed melanogenic genes in females due to higher oestrogen levels. These sex-specific genetic effects would help explain the presence of darker eye and skin pigmentation in females, as well as the well-known higher melanoma risk displayed by males.

\section{Conclusions}

Overall, the results of this work reveal the presence of sex-specific effects in human pigmentation that might be important not only in skin colour and sensitivity to sunlight but also in the higher incidence of melanoma described in males. These findings also show that, at times, sex-stratified analyses enrich genetic association studies with valuable information and knowledge.

\section{Additional file}

Additional file 1: This contains Tables S1-S3 and Figures S1-S2. Table S1. List of 363 successfully genotyped SNPs, Minor allele frequencies for all samples, males and females, and HWE $P$ value. Table S2. List of SNPS associated with pigmentation traits in females and males. Table S3. List of SNPS associated with sun response traits in females and males. Figure S1. Comparison of minor allele frequencies, female versus male individuals. Figure S2. A selection of genetic factors affecting pigmentation and sun sensitivity in humans. (PDF $417 \mathrm{~kb}$ )

\section{Abbreviations}

CEU: Utah residents with Northern and Western European ancestry;

Chr: chromosome; Cl: confidence interval; HWE: Hardy-Weinberg equilibrium; mA: minor allele; MAF: minor allele frequency; OR: odds ratio; $P$ : $P$ value; Sex-diff: sex-differentiated regression estimates test; SNP: single nucleotide polymorphism; UV: ultraviolet.

\section{Competing interests}

The authors declare that they have no competing interests.

\section{Authors' contributions}

$\mathrm{BH}, \mathrm{MI}-\mathrm{V}, \mathrm{MDB}, \mathrm{SA}, \mathrm{CM}-\mathrm{C}$ and $\mathrm{GR}$ have made substantial contributions to the conception and design and interpretation of data. BH, MI-V, LPF, MP-C, ML-C, $\mathrm{SSO}, \mathrm{CM}-\mathrm{C}$, and GR have been involved in acquisition of data and the analysis of data. BH, MI-V, LPF, MP-C, CM-C, and GR have been involved in drafting the manuscript or revising it critically for important intellectual content. All authors have given final approval of the version to be published, and all authors also agree to be accountable for all aspects of the work in ensuring that questions related to the accuracy or integrity of any part of the work are appropriately investigated and resolved. CM-C and GR conceived the study.

\section{Acknowledgements}

We thank the Madrid College of Lawyers and all patients from the different contributing Hospitals. We would like to thank Tais Moreno, M. Rosario Alonso, and Guillermo Pita for their expert technical assistance with Illumina genotyping, performed at the Spanish National Genotyping Centre (CeGen, Madrid). MI-V is funded by the "Ministry of Health Carlos III" under a Sara Borrell contract (CD15/00153). ML-C is funded by a Prometeo contract (2015/005). SSO is funded by the "Ministry of Education, Culture and Sport" under a FPU fellowship (FPU13/04976). GR is funded by the "Ministry of Health Carlos III" under a Miquel Servet II contract (CPII14-00013).

This work has also been partly funded by a research project from the Spanish Ministry of Economy and Competitiveness (CGL2014-58526-P), whose principal investigator is SA.

\footnotetext{
Author details

${ }^{1}$ Department of Medicine, Jaume I University of Castellon, Av. Sos Baynat s/n, 12071 Castellon, Spain. ²Department of Medical Oncology, Biomedical Research Institute - INCLIVA, University of Valencia, Av. Menendez Pelayo 4 accesorio, 46010 Valencia, Spain. ${ }^{3}$ Molecular Oncology and Nutritional Genomics of Cancer Group, IMDEA Food Institute, CEI UAM + CSIC, Madrid, Spain. ${ }^{4}$ Department of Genetics, Physical Anthropology and Animal Physiology, University of the Basque Country UPV/EHU, Leioa, Bizkaia, Spain. ${ }^{5}$ Department of Cell Biology and Histology, University of the Basque Country UPV/EHU, Leioa, Bizkaia, Spain. ${ }^{6}$ BioCruces Health Research Institute, Cruces University Hospital, Cruces-Barakaldo, Bizkaia, Spain.
} 
Received: 16 February 2016 Accepted: 7 March 2016

Published online: 18 March 2016

\section{References}

1. Liu F, Wen B, Kayser M. Colorful DNA polymorphisms in humans. Semin Cell Dev Biol. 2013;24:562-75

2. Simon JD, Peles D, Wakamatsu K, Ito S. Current challenges in understanding melanogenesis: bridging chemistry, biological control, morphology, and function. Pigment Cell Melanoma Res. 2009;22:563-79.

3. Norton HL, Kittles RA, Parra E, McKeigue P, Mao X, Cheng K, et al. Genetic evidence for the convergent evolution of light skin in Europeans and East Asians. Mol Biol Evol. 2007;24:710-22.

4. Jablonski NG, Chaplin G. The evolution of human skin coloration. J Hum Evol. 2000;39:57-106

5. Rijken F, Bruijnzeel PLB, van Weelden H, Kiekens RCM. Responses of black and white skin to solar-simulating radiation: differences in DNA photodamage, infiltrating neutrophils, proteolytic enzymes induced, keratinocyte activation, and IL-10 expression. J Invest Dermatol. 2004:122:1448-55.

6. Sulem P, Gudbjartsson DF, Stacey SN, Helgason A, Rafnar T, Magnusson KP, et al. Genetic determinants of hair, eye and skin pigmentation in Europeans. Nat Genet. 2007:39:1443-52.

7. Sturm RA, Teasdale RD, Box NF. Human pigmentation genes: identification, structure and consequences of polymorphic variation. Gene. 2001;277:49-62.

8. Fernandez LP, Milne RL, Pita G, Avilés JA, Lázaro P, Benítez J, et al. SLC45A2: a novel malignant melanoma-associated gene. Hum Mutat. 2008;29:1161-7.

9. Fernandez LP, Milne RL, Pita G, Floristan U, Sendagorta E, Feito M, et al. Pigmentation-related genes and their implication in malignant melanoma susceptibility. Exp Dermatol. 2009:18:634-42.

10. Duffy DL, Zhao ZZ, Sturm RA, Hayward NK, Martin NG, Montgomery GW. Multiple pigmentation gene polymorphisms account for a substantial proportion of risk of cutaneous malignant melanoma. J Invest Dermatol. 2010;130:520-8.

11. Ibarrola-Villava M, Fernandez LP, Pita G, Bravo J, Floristan U, Sendagorta E, et al. Genetic analysis of three important genes in pigmentation and melanoma susceptibility: CDKN2A, MC1R and HERC2/OCA2. Exp Dermatol. 2010;19:836-44

12. Ibarrola-Villava M, Hu H-H, Guedj M, Fernandez LP, Descamps V, Basset-Seguin N, et al. MC1R, SLC45A2 and TYR genetic variants involved in melanoma susceptibility in southern European populations: results from a meta-analysis. Eur J Cancer Oxf Engl 1990. 2012;48:2183-91.

13. Sturm RA. Molecular genetics of human pigmentation diversity. Hum Mol Genet. 2009;18:R9-17.

14. Peña-Chilet M, Blanquer-Maceiras M, Ibarrola-Villava M, Martinez-Cadenas C, Martin-Gonzalez M, Gomez-Fernandez C, et al. Genetic variants in PARP1 (rs3219090) and IRF4 (rs12203592) genes associated with melanoma susceptibility in a Spanish population. BMC Cancer. 2013;13:160.

15. Zhang M, Song F, Liang L, Nan H, Zhang J, Liu H, et al. Genome-wide association studies identify several new loci associated with pigmentation traits and skin cancer risk in European Americans. Hum Mol Genet. 2013;22:2948-59.

16. Han J, Kraft P, Nan H, Guo Q, Chen C, Qureshi A, et al. A genome-wide association study identifies novel alleles associated with hair color and skin pigmentation. PLoS Genet. 2008;4, e1000074

17. Yamaguchi $Y$, Hearing VJ. Physiological factors that regulate skin pigmentation. BioFactors Oxf Engl. 2009:35:193-9.

18. Thornton MJ. The biological actions of estrogens on skin. Exp Dermatol. 2002;11:487-502

19. Kim N-H, Cheong KA, Lee TR, Lee A-Y. PDZK1 upregulation in estrogen-related hyperpigmentation in melasma. J Invest Dermatol. 2012;132:2622-31.

20. Tadokoro T, Rouzaud F, Itami S, Hearing VJ, Yoshikawa K. The inhibitory effect of androgen and sex-hormone-binding globulin on the intracellular cAMP level and tyrosinase activity of normal human melanocytes. Pigment Cell Res. 2003;16:190-7.

21. Costin G-E, Hearing VJ. Human skin pigmentation: melanocytes modulate skin color in response to stress. FASEB J. 2007;21:976-94.

22. Kelly RI, Pearse R, Bull RH, Leveque JL, de Rigal J, Mortimer PS. The effects of aging on the cutaneous microvasculature. J Am Acad Dermatol. 1995:33:749-56.

23. Hall G, Phillips TJ. Estrogen and skin: the effects of estrogen, menopause, and hormone replacement therapy on the skin. J Am Acad Dermatol. 2005;53:555-68. quiz 569-72.
24. Martinez-Cadenas C, Peña-Chilet M, Ibarrola-Villava M, Ribas G. Gender is a major factor explaining discrepancies in eye colour prediction based on HERC2/OCA2 genotype and the IrisPlex model. Forensic Sci Int Genet. 2013;7:453-60.

25. Martinez-Cadenas C, Peña-Chilet M, Llorca-Cardeñosa MJ, Cervera R, Ibarrola-Villava M, Ribas G. Gender and eye colour prediction discrepancies: a reply to criticisms. Forensic Sci Int Genet. 2014;9:e7-9.

26. Candille SI, Absher DM, Beleza S, Bauchet M, McEvoy B, Garrison NA, et al. Genome-wide association studies of quantitatively measured skin, hair, and eye pigmentation in four European populations. PLoS One. 2012;7:e48294.

27. Joosse A, de Vries E, Eckel R, Nijsten T, Eggermont AMM, Hölzel D, et al. Gender differences in melanoma survival: female patients have a decreased risk of metastasis. J Invest Dermatol. 2011:131:719-26.

28. Roh MR, Eliades P, Gupta S, Grant-Kels JM, Tsao H. Cutaneous melanoma in women. Int J Womens Dermatol. 2015;1:21-5.

29. Laayouni H, Calafell F, Bertranpetit J. A genome-wide survey does not show the genetic distinctiveness of Basques. Hum Genet. 2010;127:455-8.

30. Ibarrola-Villava M, Fernandez LP, Alonso S, Boyano MD, Peña-Chilet M, Pita G et al. A customized pigmentation SNP array identifies a novel SNP associated with melanoma predisposition in the SLC45A2 gene. PLoS ONE. 2011;6:e19271.

31. Magi R, Lindgren CM, Morris AP. Meta-analysis of sex-specific genome-wide association studies. Genet Epidemiol. 2010;34:846-53.

32. Kocarnik JM, Park SL, Han J, Dumitrescu L, Cheng I, Wilkens LR, et al. Replication of associations between GWAS SNPS and melanoma risk in the population architecture using genomics and epidemiology (PAGE) study. J Invest Dermatol. 2014;134:2049-52.

33. Lin JY, Fisher DE. Melanocyte biology and skin pigmentation. Nature. 2007:445:843-50

34. Sulem P, Gudbjartsson DF, Stacey SN, Helgason A, Rafnar T, Jakobsdottir M, et al. Two newly identified genetic determinants of pigmentation in Europeans. Nat Genet. 2008:40:835-7.

35. Mengel-From J, Wong TH, Morling N, Rees JL, Jackson IJ. Genetic determinants of hair and eye colours in the Scottish and Danish populations. BMC Genet. 2009;10:88.

36. Nan H, Kraft P, Hunter DJ, Han J. Genetic variants in pigmentation genes, pigmentary phenotypes, and risk of skin cancer in Caucasians. Int J Cancer. 2009:125:909-17.

37. Ibarrola-Villava M, Kumar R, Nagore E, Benfodda M, Guedj M, Gazal S, et al. Genes involved in the WNT and vesicular trafficking pathways are associated with melanoma predisposition. Int J Cancer. 2015;136:2109-19.

38. Seiberg M, Paine C, Sharlow E, Andrade-Gordon P, Costanzo M, Eisinger M, et al. The protease-activated receptor 2 regulates pigmentation via keratinocyte-melanocyte interactions. Exp Cell Res. 2000;254:25-32.

39. Kempkes C, Rattenholl A, Buddenkotte J, Strozyk E, Eberle J, Hausser A, et al. Proteinase-activated receptors 1 and 2 regulate invasive behavior of human melanoma cells via activation of protein kinase D1. J Invest Dermatol. 2012;132:375-84.

40. Gudbjartsson DF, Sulem P, Stacey SN, Goldstein AM, Rafnar T, Sigurgeirsson B, et al. ASIP and TYR pigmentation variants associate with cutaneous melanoma and basal cell carcinoma. Nat Genet. 2008;40:886-91.

41. Du J, Widlund HR, Horstmann MA, Ramaswamy S, Ross K, Huber WE, et al. Critical role of CDK2 for melanoma growth linked to its melanocyte-specific transcriptional regulation by MITF. Cancer Cell. 2004;6:565-76.

42. Bakker AB, Schreurs MW, de Boer AJ, Kawakami Y, Rosenberg SA, Adema GJ, et al. Melanocyte lineage-specific antigen gp100 is recognized by melanomaderived tumor-infiltrating lymphocytes. J Exp Med. 1994;179:1005-9.

\section{Submit your next manuscript to BioMed Central} and we will help you at every step:

- We accept pre-submission inquiries

- Our selector tool helps you to find the most relevant journal

- We provide round the clock customer support

- Convenient online submission

- Thorough peer review

- Inclusion in PubMed and all major indexing services

- Maximum visibility for your research

Submit your manuscript at www.biomedcentral.com/submit 